\title{
Experiência de Uso de Jogos Educacionais Digitais Individuais em Contextos de Colaboração
}

\author{
Rachel C. D. Reis ${ }^{1,2}$, Carla L. Rodriguez ${ }^{3}$, Kamila T. Lyra ${ }^{1}$, Reginaldo Gotardo ${ }^{4}$, \\ Érica Stamato ${ }^{4}$, Seiji Isotani $^{1}$ \\ ${ }^{1}$ Instituto de Ciências Matemáticas e de Computação - Universidade de São Paulo, \\ Avenida Trabalhador São Carlense, 400 - Centro - 13566-590 - São Carlos, SP \\ ${ }^{2}$ Universidade Federal de Viçosa, Campus Rio Paranaíba \\ ${ }^{3}$ Universidade Federal do ABC (UFABC) \\ ${ }^{4}$ Cross Reality Sistemas S/A \\ rachel.reis@ufv.br, c.rodriguezdufabc.edu.br, kalyra_03@usp.br, \\ \{reginaldo, erica\}@educacross.com.br, sisotani@icmc.usp.br
}

\begin{abstract}
Digital math games designed to children of elementary school have been widely investigated by researchers of Computer on Education. Despite of important contributions to this area, we observed the lack of scientific studies that analyze the use of these games in collaborative situations supported by learning theories. Thus, this paper aims at investigating the use of Educacross platform games in four collaborative scenarios based on Distributed Cognition learning theory. The results achieved by a case study showed that the students developed other cognitive abilities (attention and interpretation) which were not expected by Educacross platform games.
\end{abstract}

Resumo. Os jogos digitais de matemática projetados para crianças do ensino fundamental têm sido largamente investigados por pesquisadores da área de Informática na Educação. Apesar das importantes contribuições, observou-se a carência de estudos científicos que analisem o uso desses jogos em situações colaborativas apoiadas por teorias de aprendizagem. Logo, este artigo tem como objetivo investigar o uso dos jogos da plataforma Educacross a partir de quatro cenários colaborativos fundamentados na teoria de aprendizagem Distributed Cognition. Os resultados obtidos por meio de um estudo de caso mostraram que os alunos desenvolveram outras habilidades cognitivas (atenção e interpretação) que não eram previstas pelos jogos da plataforma.

\section{Introdução}

Os jogos educacionais digitais têm se mostrado um valioso recurso didático-pedagógico, pois trazem comprovadas contribuições para o ensino e fixação de conteúdos de matemática para crianças do ensino fundamental [Costa et al. 2006, Silva et al. 2014]. Entretanto, o que ocorre, em geral, é que em algumas escolas os jogos educacionais digitais disponíveis apresentam conteúdos estáticos, que após serem utilizados e assimilados pelos alunos se tornam obsoletos e desinteressantes para uso posterior. 
Nesta perspectiva, duas soluções podem ser consideradas: (i) aquisição de novos jogos digitais para ensino de conteúdos específicos; (ii) adequação dos jogos digitais às diferentes situações/contextos de aprendizagem orientados por estratégias pedagógicas.

Com relação à aquisição de jogos educacionais específicos, Savi e Ulbricht (2008) e Frosi e Schlemmer (2010) discutem os desafios de se encontrar aplicativos com princípios pedagógicos que agreguem valor às aulas. Se por um lado as empresas especializadas no desenvolvimento de jogos falham nos objetivos de aprendizagem, por outro, os educadores tendem a sobrecarregá-los com requisitos pedagógicos que podem eliminar seu efeito motivador ligado à diversão e prazer. Logo, os autores enfatizam a necessidade de se encontrar o equilíbrio entre a visão dos desenvolvedores e educadores.

No que diz respeito à adequação dos jogos educacionais digitais às diferentes situações/contextos de aprendizagem, Costa et al. (2006) ressaltam a importância de desenvolvimento de mais pesquisas, que investiguem a melhor forma de trabalhar com esses recursos, ou seja, que identifiquem as melhores estratégias pedagógicas a serem utilizadas no desenvolvimento e/ou no uso dessas aplicações. Uma revisão da literatura realizada por Ribeiro et al. (2015) mostrou que, apesar do uso crescente dos jogos digitais no contexto educacional brasileiro, o desenvolvimento e aplicação desses jogos ainda estão pouco vinculados às teorias de aprendizagem. Adicionalmente, Prieto et al. (2005) destacam a importância da inserção desses jogos em contextos e situações de aprendizagem apoiadas por metodologias que orientem o processo de aprendizagem, e que promovam o desenvolvimento de novas competências e habilidades nos alunos.

Com base no exposto anteriormente, este trabalho se propõe a investigar o uso dos jogos educacionais digitais da plataforma Educacross [Educacross 2016] por meio de cenários colaborativos fundamentados na teoria de aprendizagem Distributed Cognition [Salomon 1993]. Espera-se que a partir da criação desses cenários, os alunos tenham outros ganhos de aprendizagem, além dos previstos pelos jogos da plataforma.

Além dessa seção introdutória, este trabalho está organizado da seguinte forma: na Seção 2 são apresentados os trabalhos relacionados. Na sequência, a Seção 3 apresenta o método utilizado para o desenvolvimento deste estudo. Na Seção 4 é apresentado o planejamento e desenvolvimento do estudo de caso. $\mathrm{Na}$ Seção 5 são apresentados os resultados e, em seguida, a Seção 6 analisa os ganhos de aprendizagem obtidos pelos alunos. Finalmente, na Seção 7 são apresentadas as conclusões.

\section{Trabalhos Relacionados}

A partir da revisão sistemática desenvolvida por Pietruchinski et al. (2011), observou-se que as pesquisas científicas sobre o uso de jogos educacionais digitais, em geral, destinam-se: (i) ao desenvolvimento de novos jogos para ensino de conteúdos específicos e/ou (ii) aplicação e avaliação de jogos digitais em ambientes reais de aprendizagem (e.g., sala de aula).

Os jogos educacionais digitais no contexto da área de matemática, projetados para crianças do ensino fundamental, têm sido desenvolvidos com o propósito de possibilitar que os alunos exercitem conteúdos específicos como: conjuntos numéricos [Pierini et al. 2012], operações de soma e subtração [Silva et al. 2014], construção do pensamento multiplicativo [Silveira et al. 2012], dentre outros. Para a utilização efetiva desses jogos espera-se que seja feito um planejamento adequado de como esses aplicativos serão inseridos no contexto da sala de aula, especificando, por exemplo, as 
atividades que serão utilizadas como plano de fundo para o uso dos jogos e os objetivos de aprendizagem a serem alcançados pelos alunos.

Com relação aos estudos que investigam a aplicação dos jogos educacionais digitais em contextos reais de aprendizagem [Costa et al. 2006, Gonçalves et al. 2014, Silva et al. 2014], observou-se que, na grande maioria dos casos, esses jogos estão descontextualizados em relação ao que se aprende em sala de aula.

Apesar das importantes contribuições desses trabalhos, observou-se a carência de estudos que investiguem os jogos educacionais digitais, em diferentes situações de aprendizagem, especificamente em circunstâncias colaborativas. No intuito de contribuir com pesquisas nessa área, este trabalho selecionou para investigação os jogos educacionais digitais da plataforma Educacross [Educacross 2016], que se encontra em uso pela Escola Paidéia desde o ano de 2014.

\section{Metodologia}

Seguindo a conceituação metodológica apresentada por Bogdan e Biklen (2013), este trabalho é caracterizado como uma pesquisa qualitativa de natureza básica, com objetivos de caráter descritivo, utilizando como procedimento de investigação o desenvolvimento de um estudo de caso.

Trata-se de uma pesquisa básica por investigar os benefícios de aprendizagem obtidos pelos estudantes em situações de colaboração, fundamentadas na teoria de aprendizagem Distributed Cognition [Salomon 1993], para o desenvolvimento de atividades utilizando os jogos educacionais digitais da plataforma Educacross [Educacross 2016]. Os objetivos são de caráter descritivo uma vez que o presente estudo busca identificar o desenvolvimento de outras habilidades cognitivas nos alunos, ao serem inseridos em situações de colaboração, que não são previstas pelos jogos educacionais da plataforma. Quanto aos procedimentos, o método de investigação utilizado baseia-se nos pressupostos de um estudo de caso envolvendo a análise da participação de estudantes em um contexto real de aprendizagem.

\section{Planejamento do Estudo de Caso}

\subsection{Apresentação do Contexto}

Este estudo de caso foi desenvolvido na Escola Paidéia, localizada em Bebedouro - SP. Uma parceria estabelecida entre a escola e a empresa de inovação tecnológica Cross Reality $^{I}$, possibilitou a integração dos recursos da plataforma Educacross [Educacross 2016] nas atividades realizadas pelos alunos nas aulas de informática. A plataforma Educacross, desenvolvida pela empresa Cross Reality, é composta por jogos educacionais digitais de matemática para as séries iniciais do ensino fundamental. Atualmente, os jogos disponíveis foram criados para um jogador (single-player).

$\mathrm{O}$ interesse de desenvolvimento deste estudo na Escola Paidéia originou da necessidade de investigar um ambiente real de aprendizagem em que já houvesse experiência de uso de jogos educacionais digitais pelos alunos. Dado que a plataforma Educacross é utilizada pela Escola Paidéia desde o ano de 2014, e a concordância da

\footnotetext{
${ }^{1}$ Disponível em $<$ http://crossreality.com.br/>.
} 
direção da escola para o desenvolvimento desse estudo, foram realizados três encontros na escola durante o mês de Maio/2016 com duração média de $1 \mathrm{~h}$ cada.

Com relação à seleção dos participantes, foi definido que poderiam participar do estudo crianças com idade entre 5-10 anos, visto que os jogos da plataforma Educacross foram projetados para as séries iniciais do ensino fundamental I. Dessa forma, a direção da escola selecionou uma turma do $5^{\circ}$ ano formada por 18 alunos na faixa de idade de 910 anos. Vale ressaltar que desse grupo, três alunos possuem transtorno do espectro autista $^{2}$ e não foram considerados para efeito de análise. Logo, são considerados participantes desse estudo 15 alunos, sendo nove mulheres e seis homens.

\subsection{Criação dos Cenários de Colaboração}

Para a construção dos cenários de colaboração, inicialmente, foi solicitado ao professor responsável pela turma que criasse um roteiro das atividades a ser trabalhado com os alunos na plataforma Educacross [Educacross 2016] em um determinado período. Com base no roteiro estabelecido, foram criados quatro cenários de colaboração fundamentados na teoria de aprendizagem Distributed Cognition [Salomon 1993].

De acordo com a teoria Distributed Cognition, todos os membros do grupo devem participar ativamente das resoluções dos problemas. Os benefícios de aprendizagem esperados por essa teoria contemplam: a aquisição de novos conhecimentos e o desenvolvimento de habilidades cognitivas e meta-cognitivas. Nesse artigo, além dos ganhos de aquisição de novos conhecimentos, previstos pelos jogos educacionais digitais da plataforma Educacross, espera-se ampliar o potencial pedagógico desses jogos a partir da construção de cenários colaborativos que instiguem o raciocínio dos alunos, e promovam a discussão entre seus pares, para o desenvolvimento de outras habilidades cognitivas [Salomon 1993].

Para a formação dos grupos de aprendizagem foi determinado que os membros tivessem nível similar de conhecimento sobre o conteúdo, conforme especificado pela teoria de aprendizagem Distribution Cognition. Além disso, os traços de personalidade também foram considerados no agrupamento dos estudantes ${ }^{3}$, dada a importância desse elemento para o trabalho em grupo [Reis et al. 2015].

\subsubsection{Cenário Colaborativo 1 (Atividade: Sub Sapo - níveis 4 e 5)}

- Objetivo: exercitar a resolução de operações de subtração com números inteiros de até dois dígitos.

- Material:

- Tiras de papel: criar duas histórias de quatro sentenças (uma para cada nível) com base no plano de fundo do jogo Sub sapo e dividir a história em tiras de papel (uma sentença por tira).

\footnotetext{
2 Os alunos com transtorno do espectro autista não foram considerados na análise, pois alguns instrumentos não eram adaptados a essas crianças. Além disso, os cenários de colaboração foram criados em outro nível para se adequarem ao estágio cognitivo desses alunos.

3 Este estudo é um recorte de um trabalho maior, no âmbito de um projeto de doutorado, em desenvolvimento desde o ano de 2014 no contexto de formação de grupos e traços de personalidade. Embora os traços de personalidade tenham sido considerados para o agrupamento dos estudantes, nesse em artigo, em particular, a análise dos resultados não levará em conta esse elemento.
} 
V Congresso Brasileiro de Informática na Educação (CBIE 2016)

Anais do XXII Workshop de Informática na Escola (WIE 2016)

o Folhas de resposta em papel: criar questões que instiguem o raciocínio dos alunos. As perguntas devem estar associadas aos elementos das operações matemáticas mostradas no jogo.

o Tablet: exibir o jogo Sub sapo na plataforma Educacross.

- Procedimento:

Antes de iniciar o jogo, cada grupo deve receber: i) uma folha de resposta, ii) as tiras de papel, e iii) um tablet. O procedimento para desenvolvimento das atividades do jogo educacional Sub sapo é ilustrado pela Figura 1 e consiste nos seguintes passos:

1. Colocar as tiras em ordem para que a história faça sentido e numerá-las (Figura 1.a);

2. Ler as perguntas apresentadas na folha de respostas (Figura 1.b);

3. Relacionar os elementos da operação matemática à história e identificar o que cada número mostrado no jogo representa (Figura 1.c);

4. Responder as perguntas apresentadas na folha de respostas (Figura 1.d);

5. Selecionar uma opção na plataforma Educacross para o jogo Sub sapo (Figura 1.e).

5.1. No caso de resposta incorreta, os alunos devem repetir os passos 3 e 4.

6. Repetir os passos 3, 4 e 5 até que encerrem as operações do jogo Sub sapo.

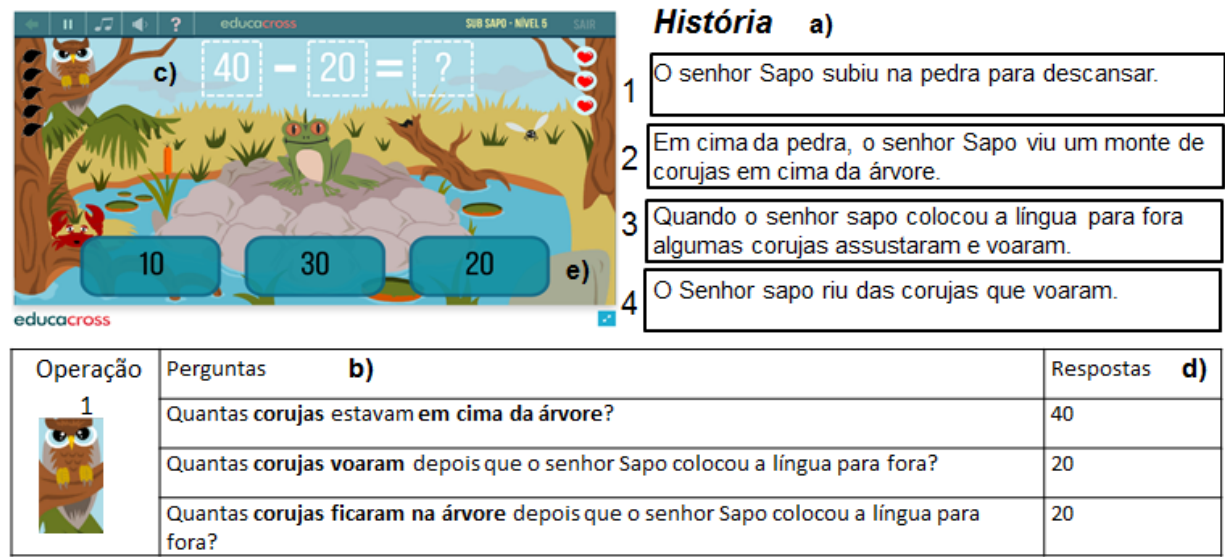

Figura 1. Exemplo de cenário colaborativo para o desenvolvimento das atividades do jogo Sub sapo - níveis 4 e 6. Fonte: [Educacross 2016].

\subsubsection{Cenários Colaborativos 2 (Super heroína - nível 1) e 3 (Mini herói - nível 4)}

- Objetivo: exercitar a resolução de operações de subtração com números inteiros de até três dígitos (cenário 2); exercitar a identificação dos operadores de soma, subtração, divisão e multiplicação em operações de até três dígitos (cenário 3).

- Material:

o Descrição do desafio em papel: criar um desafio com base no plano de fundo do jogo Super heroína (cenário 2) ou Mini herói (cenário 3) e deixar um espaço para que os alunos possam desenvolver o cálculo da operação.

- Tablet: exibir o jogo Super heroína (cenário 2) ou Mini herói (cenário 3) na plataforma Educacross.

- Procedimento

Antes de iniciar os jogos, cada grupo deve receber: i) instruções do desafio em papel e ii) um tablet. O procedimento para desenvolvimento das atividades do jogo Super heroína é ilustrado pela Figura 2 e consiste nos seguintes passos: 
V Congresso Brasileiro de Informática na Educação (CBIE 2016)

Anais do XXII Workshop de Informática na Escola (WIE 2016)

1. Ler o desafio (Figura 2.a);

2. Para cada operação:

2.1. Um membro deve manusear o tablet com o jogo da Super heroína (Figura 2.b);

2.2. O outro membro deve utilizar o espaço disponibilizado na folha em papel para efetuar o cálculo da operação (Figura 2.c);

2.3. Na operação seguinte, o tablet e a folha em papel devem ser trocados entre os membros do grupo;

2.4. Repetir os passos 2.1, 2.2, 2.3 até que finalizem as operações do jogo.

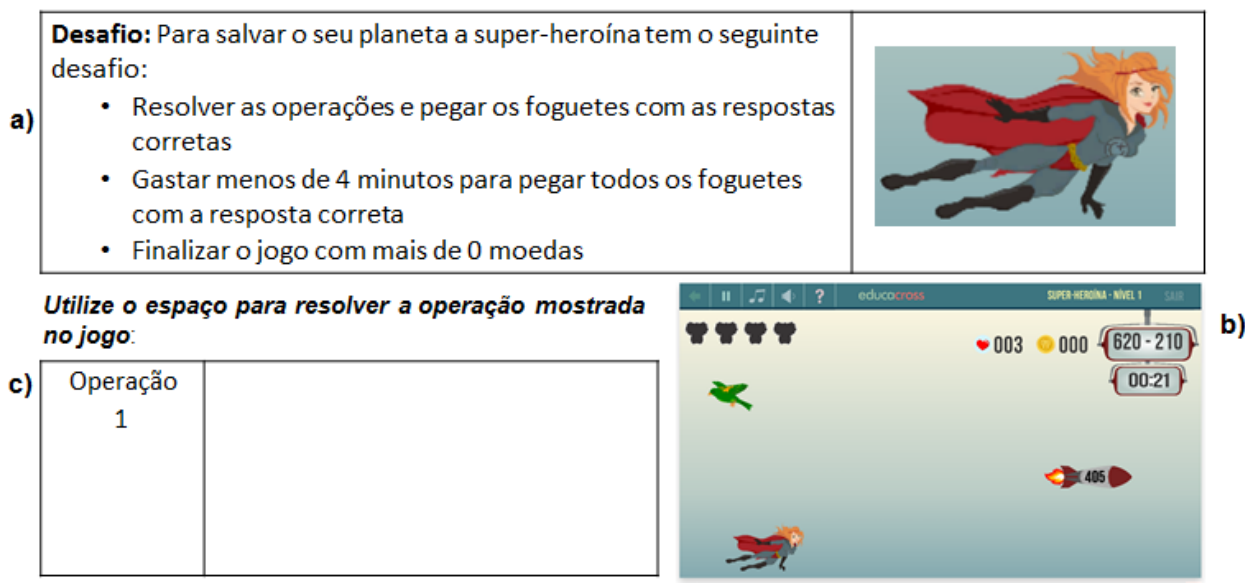

Figura 2. Exemplo de cenário colaborativo para o desenvolvimento da atividade do jogo Super heroína - nível 1. Fonte: [Educacross 2016].

Para o cenário 3 (jogo Mini Herói), o procedimento para desenvolvimento das atividades segue a mesma sequência de passos mostrada para o jogo da Super heroína.

\subsubsection{Cenário Colaborativo 4 (Atividade: Soma sapo - níveis 4, 5 e 6)}

- Objetivo: exercitar a resolução de operações de soma com números inteiros de até dois dígitos.

- Material:

o Folhas de resposta em papel: elaborar três histórias (uma para cada nível) com base no plano de fundo do jogo Soma sapo e criar questões que instiguem o raciocínio dos alunos. As perguntas devem estar associadas aos elementos das operações matemáticas mostradas no jogo.

o Tablet: exibir o jogo Soma sapo na plataforma Educacross.

- Procedimento:

Antes de iniciar o jogo, cada grupo deve receber: i) uma folha de resposta, e iii) um tablet. O procedimento para desenvolvimento das atividades do jogo Soma sapo é ilustrado pela Figura 3 e consiste nos seguintes passos:

1. Ler a história (Figura 3.a) e as perguntas na folha de respostas (Figura 3.b);

2. Relacionar os elementos da operação matemática à história e identificar o que cada número mostrado no jogo representa (Figura 3.c);

3. Responder as perguntas apresentadas na folha de respostas (Figura 3.d);

4. Selecionar a opção no jogo Soma sapo (Figura 3.e).

4.1. No caso de resposta incorreta, os alunos devem repetir os passos 3 e 4 . 
V Congresso Brasileiro de Informática na Educação (CBIE 2016)

Anais do XXII Workshop de Informática na Escola (WIE 2016)

5. Repetir os passos 2, 3 e 4 até que encerrem as operações no jogo Soma sapo.

a)

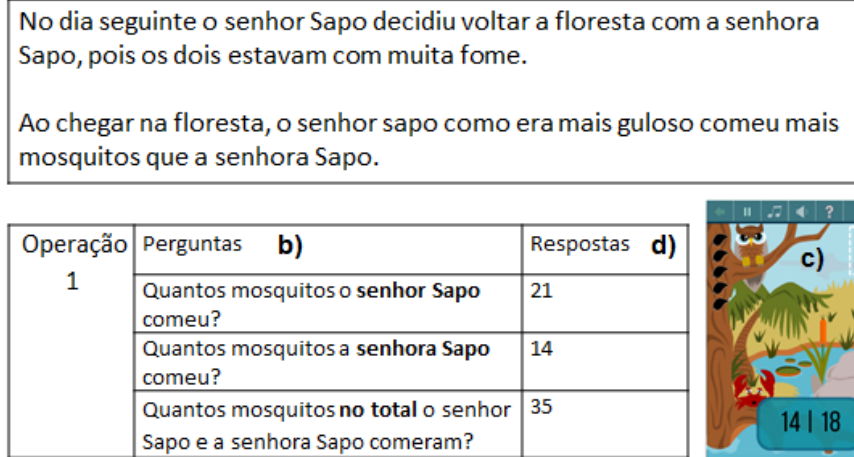

\begin{tabular}{|c|l|l|}
\hline Operação & Perguntas b) & Respostas d) \\
\cline { 2 - 3 } 1 & $\begin{array}{l}\text { Quantos mosquitos o senhor Sapo } \\
\text { comeu? }\end{array}$ & 21 \\
\cline { 2 - 3 } & $\begin{array}{l}\text { Quantos mosquitos a senhora Sapo } \\
\text { comeu? }\end{array}$ & 14 \\
\cline { 2 - 3 } & $\begin{array}{l}\text { Quantos mosquitos no total o senhor } \\
\text { Sapo e a senhora Sapo comeram? }\end{array}$ & 35 \\
\hline
\end{tabular}

Figura 3. Exemplo de cenário colaborativo para o desenvolvimento da atividade do jogo Soma sapo - nível 6. Fonte: [Educacross 2016].

\subsection{Instrumentos}

$\mathrm{Na}$ coleta de dados foram utilizados instrumentos para análise individual do aluno e para análise do grupo. O primeiro instrumento de análise individual é composto por três testes de conhecimento para avaliar o quanto o aluno sabe sobre as operações de soma e subtração em três etapas diferentes: uma semana antes da atividade em grupo (pré-teste), logo após encerrar a atividade em grupo (pós-teste) e uma semana depois de realizada a atividade em grupo (teste de retenção). O segundo instrumento utilizado foi o questionário psicológico ETPC (Escala de Traços de Personalidade para Crianças) [Sisto 2004], para identificar os traços de personalidade dos alunos ${ }^{4}$. Com relação aos instrumentos para análise do grupo, foram utilizados um roteiro de observação e três câmeras filmadoras para registrar o comportamento dos alunos durante a interação.

\subsection{Etapas}

O estudo de caso apresentado neste trabalho foi realizado em três etapas:

1. Na etapa 1 (pré-teste), os alunos foram submetidos à resolução de 2 testes individuais em papel: pré-teste de conhecimento e teste de personalidade. O primeiro teste, composto por 4 exercícios, identificou o conhecimento inicial dos alunos sobre as operações de soma e subtração de 2 e 3 dígitos. O segundo utilizou o questionário ETPC para identificar os traços de personalidade de cada um dos participantes.

2. Na etapa 2 (intervenção + pós-teste), os alunos foram divididos em seis duplas e um trio. Na sequência, os grupos foram submetidos aos 4 cenários de colaboração descritos na Seção 4.2. Após a interação, os participantes responderam individualmente ao pós-teste de conhecimento composto por 3 questões envolvendo as operações de soma e subtração de até 3 dígitos e um exercício de interpretação.

3. Na etapa 3 (teste de retenção), os alunos responderam a um teste individual de retenção, no intuito de avaliar o conhecimento fixado pelos alunos, composto por 3 questões envolvendo as operações de soma e subtração de até três dígitos e um exercício de interpretação.

\footnotetext{
${ }^{4}$ Conforme mencionado anteriormente, a análise dos traços de personalidade dos estudantes não será realizada neste trabalho.
} 
V Congresso Brasileiro de Informática na Educação (CBIE 2016)

Anais do XXII Workshop de Informática na Escola (WIE 2016)

\section{Resultados}

A Tabela 1 apresenta um resumo dos resultados obtidos a partir da análise dos testes de conhecimento: pré-teste, pós-teste e teste de retenção.

Tabela 1 - Percentual dos principais erros e resultados em branco nos testes de conhecimento.

\begin{tabular}{|c|c|c|c|c|c|}
\hline $\begin{array}{c}\text { Teste de } \\
\text { Conhecimento }\end{array}$ & Operações & $\begin{array}{c}\text { Confusão no uso dos } \\
\text { operadores +e - }\end{array}$ & $\begin{array}{c}\text { Erros de } \\
\text { cálculo }\end{array}$ & $\begin{array}{c}\text { Respostas em } \\
\text { branco }\end{array}$ & $\begin{array}{c}\text { Erro de } \\
\text { interpretação }\end{array}$ \\
\hline \multirow{2}{*}{ Pré-teste } & Soma & $20 \%$ & $20 \%$ & - & N/A \\
\cline { 2 - 6 } & Subtração & $60 \%$ & $20 \%$ & $13,33 \%$ & N/A \\
\hline \multirow{2}{*}{ Pós-teste } & Soma & - & - & - & N/A \\
\cline { 2 - 6 } & Subtração & $46,67 \%$ & - & - & $40 \%$ \\
\cline { 2 - 6 } & Soma & - & - & - & N/A \\
\cline { 2 - 6 } & Subtração & $60 \%$ & $6,67 \%$ & $6,67 \%$ & $40 \%$ \\
\hline
\end{tabular}

De acordo com a Tabela 1, os erros cometidos pelos alunos nos testes de conhecimento foram divididos em "confusão no uso dos operadores $(+)$ e (-)" (coluna 3), "erros de cálculo" (coluna 4), e "erro de interpretação" (coluna 6). Além disso, a Tabela 1 também mostra o percentual de "respostas deixadas em branco" (coluna 5).

No pré-teste, observou-se que o erro de "confusão no uso dos operadores (+) e (-)" (coluna 3) ocorreu com maior frequência $(60 \%$ - 9 alunos) nas operações de subtração para identificar o valor do $1^{\circ}$ ou $2^{\circ}$ operando. Os erros mais comuns foram:
Ex.1) $65-?=13 \rightarrow 78$
Ex.2) $80-?=77 \rightarrow 157$
Ex.3) $?-5=80 \rightarrow 75$

No caso da operação de soma, foi observado que $20 \%$ (3 alunos) trocou o sinal de $(+)$ por (-) no momento de efetuar o cálculo, conforme mostrado nos exemplos abaixo:
Ex1) $730+40=690$
Ex.2) $505+302=203$
Ex.3) $99+14=85$

Com relação aos "erros de cálculo" (coluna 4) no pré-teste, a ocorrência foi de $20 \%$ (3 alunos), tanto nas operações de soma quanto nas operações de subtração. No caso das "respostas em branco" (coluna 5), a ocorrência foi de 13,33\% (2 alunos).

No pós-teste, os 15 alunos acertaram todas as operações de soma. No que diz respeito às operações de subtração, apesar da redução do número de alunos que cometeram o erro de "confusão no uso dos operadores (+) e (-)" (coluna 3) do pré-teste para o pós-teste, foi observado que 46,67\% (7 alunos) continuou cometendo esse tipo de erro, sendo que $40 \%$ dos alunos errou o resultado quando o sinal de interrogação (?) foi colocado no lugar do $1^{\circ}$ operando (e.g., ? - $5=80 \rightarrow 75$ ), e $6,67 \%$ ( 1 aluno) errou quando o sinal de interrogação (?) foi colocado no lugar do $2^{\circ}$ operando (e.g., 65 - ? = $13 \rightarrow 78$ ). Os "erros de cálculo" (coluna 4) e "respostas em branco" (coluna 5), não ocorreram no pós-teste. Com relação ao "erro de interpretação" (coluna 6), 40\% (6 alunos) não conseguiu relacionar a operação às questões apresentadas no exercício.

No teste de retenção os 15 alunos acertaram todas as operações de soma. Por outro lado, o erro de "confusão no uso dos operadores (+) e (-)" (coluna 3)" voltou a ser cometido por $60 \%$ ( 9 alunos). Desse grupo, $46,67 \%$ ( 7 alunos) errou o resultado quando o sinal de interrogação (?) foi colocado no lugar do $1^{\circ}$ operando (e.g., ? - $13=33 \rightarrow 20$ ) e 13,33\% (2 alunos) errou quando o sinal de interrogação (?) foi colocado no $2^{\circ}$ operando (e.g., 37 - ? = $13 \rightarrow$ 50). Os "erros de cálculo" (coluna 4) foram cometidos por $6,67 \%$ ( 1 aluno) e os "erros de interpretação" (coluna 6) por 40\% (6 alunos). Por fim, foi constatado que $6,67 \%$ (1 aluno) deixou operações de subtração sem resposta ("resposta em branco" - coluna 5). 
V Congresso Brasileiro de Informática na Educação (CBIE 2016)

Anais do XXII Workshop de Informática na Escola (WIE 2016)

\section{Análise e Discussão dos Resultados}

Essa seção apresenta uma análise dos ganhos de aprendizagem obtidos pelos estudantes ao serem inseridos nos cenários de colaboração apresentados na Seção 4.2, utilizando os jogos educacionais digitais da plataforma Educacross [Educacross 2016].

A partir da Tabela 1, é possível observar que os ganhos de aprendizagem de "aquisição de novos conhecimentos" previstos pela plataforma Educacross foram alcançados pelos alunos. De acordo com a Tabela 1, os erros de "confusão no uso dos operadores (+) e (-)" (coluna 3), e "erros de cálculo" (coluna 4) identificados no préteste para as operações de soma, foram completamente resolvidos no pós-teste e teste de retenção. Com relação às operações de subtração, apesar dos erros de "confusão de uso dos operadores (+) e (-)" (coluna 3) terem persistido no pós-teste e teste de retenção (coluna 3), pode-se verificar a redução dos "erros de cálculo" (coluna 4) nas operações de subtração. Esses resultados vão de encontro com os objetivos pedagógicos dos jogos da plataforma Educacross quando utilizados individualmente (single-player).

Com relação à ampliação do potencial pedagógico dos jogos da plataforma Educacross ao serem inseridos nos cenários colaborativos apresentados na Seção 4.2, foi possível observar o desenvolvimento de outras habilidades cognitivas nos alunos como atenção e interpretação. A habilidade cognitiva de atenção foi percebida com base na redução dos "erros de cálculo" (Tabela 1, coluna 4) nas operações de soma e subtração. A habilidade de interpretação foi verificada em $60 \%$ da turma (8 alunos) que resolveu corretamente o exercício de interpretação no pós-teste e teste de retenção. Esses resultados estão de acordo com a teoria de aprendizagem Distributed Cognition que prevê o desenvolvimento desse tipo de habilidade (cognitiva) nos alunos.

Os resultados apresentados mostram indícios de que é possível desenvolver outras habilidades cognitivas nos estudantes, que não sejam previstas pelos jogos educacionais digitais, por meio da criação de cenários de colaboração apoiados por teorias de aprendizagem. Considerando que existem diversas teorias de colaboração com diferentes objetivos de aprendizagem, é possível adotar novas estratégias pedagógicas para a construção de cenários com a proposta de se obter outros benefícios.

\section{Conclusão}

Este trabalho investigou o uso dos jogos educacionais da plataforma Educacross por meio de cenários colaborativos fundamentados na teoria de aprendizagem Distributed Cognition. Essa investigação foi feita por um estudo de caso envolvendo 15 crianças do ensino fundamental I. Os resultados mostraram que além da aquisição de conhecimento prevista pelos jogos da plataforma, os cenários de colaboração contribuíram para o desenvolvimento de habilidades cognitivas relacionadas à atenção e interpretação.

Com base nos resultados obtidos observa-se que os jogos educacionais digitais podem ser utilizados em diferentes cenários de aprendizagem, entre esses os de colaboração, não havendo a necessidade da escola adquirir tecnologias específicas para o desenvolvimento de outras habilidades cognitivas. Além disso, a investigação sobre a criação de cenários que utilizem os jogos educacionais digitais em diferentes contextos pode ser útil também às empresas de desenvolvimento, que têm a oportunidade de incorporar novos elementos à dinâmica de seus jogos. No caso dos cenários criados para os jogos da plataforma Educacross, sugere-se, por exemplo, a incorporação de uma narrativa e/ou um contexto aos jogos, desafios de interpretação e elementos de feedback. 
V Congresso Brasileiro de Informática na Educação (CBIE 2016)

Anais do XXII Workshop de Informática na Escola (WIE 2016)

A proposta é que essas ações levem os alunos à reflexão sobre o conteúdo e erros cometidos, instigando seu raciocínio para a resolução das operações de forma adequada.

Como trabalho futuro, sugere-se a ampliação dos experimentos envolvendo maior número de estudantes, bem como a criação de cenários mais específicos com o suporte de teorias de aprendizagem mais adequadas para resolver, por exemplo, os erros de "confusão no uso dos operadores (+) e (-)" (Tabela 1 - coluna 3) para identificar o valor de um dos operandos nas operações de subtração (e.g., ? - $13=33 \rightarrow 20$ ).

\section{Agradecimentos}

Os autores agradecem ao CNPq pelo apoio financeiro e a colaboração da Escola Paidéia, em especial, da coordenadora Neli Volpini e das professoras Patrícia e Léia.

\section{Referências}

Bogdan, R. and Biklen, S. (2013). Investigação Qualitativa em Educação - Uma introdução à teoria dos métodos, (M. J. Alvarez, S. S. B. Santos \& T. M. Baptista, Trads). Porto Editora.

Costa, J. R. M., Soares, A. B. and Lima, C. (2006). "Jogar a Aprender: a Informática no Ensino de Algebra Elementar", XVII Simpósio Brasileiro de Informática na Educação, p. 81-90.

Educacross (2016). "A plataforma da Educação Inteligente", Disponível em $<$ https://www.educacross.com.br/ $>$. Acesso em 08 de junho de 2016.

Frosi, F. O. and Schlemmer, E. (2010). "Jogos Digitais no Contexto Escolar: desafios e possibilidades para a Prática Docente”, IX SBGames, p. 115-122.

Gonçalves, A., Souza, M., Silva, R., Silva, D., Bueno, P., Balthazar, G. (2014). "Desenvolvimento de Jogos Educacionais na Área de Matemática em Escola de Ensino Fundamental", XIX Congresso Internacional de Informática Educativa, p. 622 - 627.

Pierini, L. M., Valentim, M. A. C., Cardoso, A. (2012). "Brinquedos Numéricos: um jogo para o ensino dos conjuntos numéricos", Simpósio Brasileiro de Informática na Educação, p. 1-8.

Pietruchinski, M. H., Neto, J. C., Malucelli, A., Reinehr, S. (2011). "Os jogos educativos no contexto do SBIE: uma revisão sistemática de Literatura”, XXII SBIE, p. 476-485.

Prieto, L. M., Trevisan, M. do C. B., Danesi, M. I., Falkembach, G. A. M. (2005). Uso das Tecnologias Digitais em Atividades Didáticas nas Séries Iniciais. Revista Renote, 3(1):1-11.

Reis, R. C. D., Rodriguez, C. L., Lyra, K. T., Jaques, P. A., Bittencourt, I. I., Isotani, S. (2015). Estado da Arte sobre Afetividade na Formação de Grupos em Ambientes Colaborativos de Aprendizagem, Revista Brasileira de Informática na Educação, 23: 113-130.

Ribeiro, R. J., Junior, N. S., Frasson, A. C., Pilatti, L. A., Silva, S. de C. R. da (2015). Teorias de Aprendizagem em Jogos Digitais Educacionais: um Panorama Brasileiro. Revista Novas Tecnologias na Educação (Renote), 13(1): 1-10.

Salomon, G. (1993). Distributed Cognitions, Cambridge University Press.

Savi, R., Ulbricht, V. R. (2008). Jogos Digitais Educacionais: Benefícios e Desafios. Revista Novas Tecnologias na Educação (Renote), 6(1): 1-10.

Silva, B. C. da, Silva, P. P., Luz, L. P. da; Silva, E. G., Martins, H. P. (2014). "Jogos digitais como instrumento didático no processo ensino-aprendizagem das operações básicas de matemática”, XXV Simpósio Brasileiro de Informática na Educação, p. 682-691.

Silveira, S. R., Rangel, A. C. S., Ciríaco, E. de L. (2012). "Utilização de Jogos Digitais para o Desenvolvimento do Raciocínio Lógico-Matemático", \#Tear: Revista de Educação, Ciência e Tecnologia, 1(1):1-14.

Sisto, F. F. (2004). Escala de Traços de Personalidade para Crianças, $1^{\text {a }}$ ed. SP: Vetor. 